Revue d'histoire de l'Amérique française

ZAS REVUE D.HISTOIRE DE L'AMÉRIQUE FRANÇAISE

\title{
La démission du Dr Meilleur comme surintendant de l'éducation (1855)
}

\section{Thomas Charland}

Volume 24, numéro 4, mars 1971

URI : https://id.erudit.org/iderudit/303018ar

DOI : https://doi.org/10.7202/303018ar

Aller au sommaire du numéro

Éditeur(s)

Institut d'histoire de l'Amérique française

ISSN

0035-2357 (imprimé)

1492-1383 (numérique)

Découvrir la revue

Citer cet article

Charland, T. (1971). La démission du Dr Meilleur comme surintendant de l'éducation (1855). Revue d'histoire de l'Amérique française, 24(4), 513-525. https://doi.org/10.7202/303018ar d'utilisation que vous pouvez consulter en ligne.

https://apropos.erudit.org/fr/usagers/politique-dutilisation/ 


\section{LA DEMISSION DU DR MEILLEUR COMME SURINTENDANT DE L'EDUCATION (1855)}

Thomas Charland, O.P.

Le 19 juin 1855, le $\mathrm{Dr}$ Jean-Baptiste Meilleur écrivait au gouverneur général pour présenter sa démission comme surintendant de l'Education du Bas-Canada et solliciter un autre emploi. Le 28, une lettre du secrétaire provincial, GeorgeEtienne Cartier, l'informait que sa démission avait été acceptée. Peu de jours après, il confiait à son ami l'abbé Louis-Edouard Bois, curé de Maskinongé:

Je suis dans une confusion extrême, étant sur le point d'abandonner la direction de l'instruction publique pour celle du bureau de poste de la cité de Montréal ! ! Le tout s'est fait cependant en vertu d'une bonne entente; mais au fond de tout cela est, d'une part, le désir d'alléger mon travail, et, de l'autre, de faire de mon département un instrument politique et surtout de plaire aux gens de Québec.1

Mes amis, ici, disent que les gens de Québec ont réussi à m'expulser et à s'emparer de mon département. C'est à peu près vrai; mais j'en suis sorti de bon gré, à la vue de la tourmente que je voyais sans cesse occupée à me dénigrer, à déprécier mes actes et à me faire des embarras. Oui, des embarras, et plus que des embarras, de la part de ceux sur la coopération empressée desquels je croyais avoir le droit de compter dans l'intérêt de la chose dont j'étais chargé. Sicotte n'a-t-il pas converti son comité en véritable inquisition? N'a-t-il pas soustrait tout ce qu'on lui a envoyé de favorable à mon administration? N'a-t-il pas soustrait mes rapports ? Chauveau n'en a-t-il pas fait autant ? N'a-t-il pas soustrait aussi mon projet de loi ? Etc. N'a-t-on pas pris les fonds consacrés à l'objet de la loi d'éducation pour faire des octrois spéciaux, et diminué ainsi, au lieu de l'augmenter, le fonds des écoles? Je puis dire que j'ai été persécuté depuis quelques années, au lieu d'être aidé, comme j'aurais dû l'être, par ceux qui étaient en position de le faire. On a même apparemment récompensé mes opposants, en leur donnant des marques de considération, et même des emplois lucratifs: voire votre éteignoir en chef *, Sicotte, Armstrong, etc., etc. Mais en voilà assez, et ce serait bien trop, si je ne parlais à vous.

130 juin 1855.

* Joseph-Edouard Turcotte, député de Maskinongé.

[ 513 ]

RHAF, vol. 24, no 4 (mars 1971) 
Que le tout soit donc strictement entre nous. Il est de ces peines qui ne doivent être connues que de Dieu et des saints auxquels les affligés se recommandent. ${ }^{2}$

Essayons de lever un peu le voile, ou plutôt - car il est passablement levé - de donner les explications nécessaires à l'intelligence de ces aveux, en utilisant d'autres lettres adressées par le Dr Meilleur à l'abbé Bois. ${ }^{3}$

La loi d'éducation du 9 juin 1846 avait rétabli, pour le soutien des écoles élémentaires, la contribution obligatoire, basée sur l'évaluation municipale. L'impopularité de cette mesure n'avait pas arrêté le Dr Meilleur. "Le projet, écrivait-il, rencontrera de l'opposition de la part de quelques membres faibles et esclaves de l'opinion populaire. Dans tous les cas, il est impossible de plaire à tout le monde, et, quant à moi, il y a longtemps que j'ai fait à cet égard mon sacrifice; je ferai ce que je considère mon devoir envers la génération naissante, advienne que pourra." " Il se forma une opposition à la loi qui alla sans cesse en grandissant et qui dégénéra, en certaines parties de ia Province, en de véritabiles petites jacqueries. Ces tristes épisodes sont passés à l'histoire sous le nom de guerre des éteignoirs.

Tenu par ses attributions de s'occuper du fonctionnement de la loi, le surintendant fut bientôt débordé. Son bureau ne désemplissait pas de gens venus lui faire part de leurs difficultés, et il n'arrivait pas, avec ses deux seuls assistants, à répondre aux lettres qui déferlaient sans cesse sur sa table. "Les éteignoirs réussiront peut-être à m'éteindre, avouait-il. Toujours est-il vrai que l'opposition qu'ils font faire à l'acte des écoles me donne un travail et une sollicitude insurmontables. Je me flatte pourtant du succès et de survivre à toutes leurs menées, sourdes ou ouvertes." 5 Il en contracta un mal de côté qui le fit souffrir beaucoup, "au point d'être quelquefois un véritable martyre".

22 juillet 1855.

3 La correspondance Meilleur-Bois fait partie de la Collection Bois, qui se trouve aujourd'hui au Grand Séminaire de Nicolet. L.-P. Audet, "Index analytique du Mémorial de l'Education dans le Bas-Canada du Dr JeanBaptiste Meilleur", Mémoires de la Société Royale du Canada, 4e série, tome 2, section I, 1964: 52. Une centaine (117) de ces lettres en avaient été distraites en faveur du P. Joseph Grenier, s.j. Elles sont aux Archives des Jésuites, à Saint-Jérôme, Québec. Le P. Léon Pouliot, s.j. en a donné un aperçu dans le Bulletin des Recherches historiques 43 (1937): 337-343.

46 mai 1846.

520 octobre 1846.

68 février 1847. 
Les élections générales de 1848 consacrèrent le triomphe de la loi sur la politique des éteignoirs. Ceux-ci furent "ignominieusement balayés des hustings comme on balaie certaines vilaines choses avec les plus mauvais balais". ${ }^{7}$ Le surintendant s'en réjouit; mais les éteignoirs redoublèrent d'acharnement contre la loi. Soulevés par ces charlatans politiques, les habitants en vinrent à des voies de fait. Au cours de l'hiver de 1850 , ils s'en prirent aux estimateurs municipaux et aux commissaires d'écoles, tondirent la crinière de leurs chevaux et mirent le feu à leurs bâtiments. Ils brisèrent les fenêtres des écoles, incendièrent même quelques-unes de ces maisons. On leur représentait que la taxe scolaire n'était qu'un commencement et qu'ils en seraient bientôt réduits aux tristes conditions qui avaient forcé les Irlandais à émigrer. Et c'est précisément dans les milieux mêlés d'immigrants irlandais que ces sinistres exploits furent perpétrés.

Le Dr Meilleur écrivait à son ami: "Les agitateurs et leurs dupes viendront, j'espère, à se fatiguer de tout cela. Pour moi, j'en mourrai peut-être à la peine." 8 Les visites de persuasion qu'il fit dans les paroisses agitées servirent de prétexte aux opposants pour le calomnier. "On persuade aux habitants que c'est la crainte de perdre mes émoluments qui me fait agir ainsi, ou la promesse d'une récompense à tant par paroisse, si je peux en gagner les habitants et les faire se soumettre à la loi. Maintenant on commence à s'organiser de manière à ne pas me laisser parler." ${ }^{\circ}$

Voyant ses efforts de persuasion contrariés, il favorisa l'emploi de la manière forte, solution qui, il faut le dire, s'accordait mieux avec son tempérament. "Ce qu'il faut sur les lieux, écrivit-il alors, ce n'est pas le surintendant de l'Education, mais une police armée, une police générale, et je ne désespère pas d'obtenir quelque secours de la sorte pour soumettre à la loi nos habitants égarés par de mauvais sujets qui spéculent sur leur ignorance en avivant leurs préjugés... Des mesures sont prises pour arrêter les émeutiers là où ils se montreront. Mais ce ne sont pas les plus coupables qu'on atteindra, mais seulement leurs dupes." 10

$71^{\text {er }}$ février 1848.

85 février 1850 .

921 février 1850 .

1021 février 1850. Trois ans plus tard (le 14 mars 1853), Meilleur écrira pour se justifier des attaques d'un certain Rusticus: "Ce n'est pas le surintendant qui a demandé de mettre sur pied la police montée. On l'a fait sans son concours; mais il fallait bien faire quelque chose pour empêcher les émeutiers, conduits par Allison, de St-Grégoire, et alimentés 
Au sortir d'une maladie qu'il avait contractée dans ses visites et qui l'immobilisa pendant quelque temps, il se sentait ruiné de toute manière, financièrement comme physiquement, au point d'être incapable de faire honneur à certains engagements qu'il avait pris. "Quant à ma contribution au collège de St-Hyacinthe, je crois que les zélés entrepreneurs auront à s'en passer, vu que je suis plus obligé à ma peau qu'à ma chemise. Je n'ai pas le moyen de faire mes propres affaires, ni véritablement celui de payer une souscription semblable, que j'ai faite dans un temps où j'avais plus d'espérance que de moyens. Maintenant je n'ai l'un ni les autres, si nécessaires pour se montrer comme je devrais pouvoir le faire en qualité de surintendant de l'Instruction publique." 11

La loi de 1841 pourvoyait à la nomination d'un seul surintendant pour tout le pays. L'année suivante, il fut convenu de lui donner deux assistants, l'un pour le Haut et l'autre pour le Bas-Canada, qui se partageraient le salaire de $£ 750$. La loi du 29 mai 1845 créa un surintendant distinct pour le BasCanada et porta son salaire de $£ 375$ à $£ 500$. Pendant trois ans, de 1842 à 1845, le Dr Meilleur dut se contenter du salaire d'un commis de bureau. Pour subvenir aux besoins rigoureux de sa famille et satisfaire aux convenances de sa position, il fut obligé de sacrifier les $£ 600$ qu'il avait retirées de la vente de ses propriétés foncières à L'Assomption et qu'il destinait à l'achat de la seigneurie de Repentigny en vue d'assurer sa subsistance pendant ses vieux jours. Il dut même s'endetter pour la somme de $£ 144$.

En vain réclama-t-il d'être traité au moins aussi bien que l'assistant d'un chef de bureau. La politique n'y trouvait pas son compte.

Voyez-vous, je ne suis pas un homme politique, et mon bureau n'exerce aucune influence politique; et la politique, grand Dieu, c'est tout le mérite, c'est tout l'homme, machiavélique ou non! Le pays se trouvera bien honoré, je suppose, d'après la manière de voir de certains hommes, qui n'en ont jamais trop pour eux, de voir, avant qu'il soit peu, peutêtre, les enfants du surintendant avoir pour unique héritage le parchemin de leur père, avec ses pendants de misère et de sollicitudes.

par les Irlandais de Ste-Monique, de brûler les propriétés. Voyant ce moyen de répression en opération, le surintendant a recommandé de le continuer; et le mal n'est pas de l'avoir employé, mais de l'avoir discontinué pour y revenir plus tard, après avoir laissé les gens se moquer et du gouvernement et de la loi; du surintendant, pas que je sache."

115 avril 1850. 
Pour moi, sachant que plus d'un ami serait heureux de me recueillir, je me retirerais demain de la vie publique, si une nombreuse famille ne me disait pas: "Papa, restez-y encore, afin de nous procurer du pain pendant que nous sommes encore trop jeunes et trop faibles pour pouvoir compter sur nos propres efforts." 12

C'est la première fois que le Dr Meilleur parle de se retirer. L'idée va l'obséder sans cesse par la suite.

En acceptant la charge d'assistant-surintendant, en 1842, il avait eu la promesse de sir Charles Bagot que le département de l'Education échapperait à l'influence politique. Mais les politiciens ne se résignèrent pas à abandonner cette source de patronage, qui augmentait d'année en année.

$\mathrm{Au}$ printemps de 1851, il fut question de faire du département un ministère et d'adjoindre au surintendant-ministre deux députés-surintendants, l'un pour le district de Québec et l'autre pour celui de Montréal. Meilleur s'opposa fermement à la proposition d'un surintendant-ministre. Le titulaire pourrait être un ignorant - n'avait-on pas vu un pauvre charpentier du Haut-Canada nommé arpenteur général, en 1841 ? De toute façon, il n'inspirerait pas confiance, n'offrant pas de garantie d'impartialité. ${ }^{13}$ Il était prêt, quant à lui, à accepter des députéssurintendants, pourvu qu'ils ne fussent pas des créatures politiques et qu'ils ne fussent pas payés à même le fonds des écoles. Mais, comme les auteurs du projet n'étaient pas de ses amis Joseph Cauchon l'avait maltraité dans son Journal de Québec, et Jacques Crémazie avait soulevé contre lui les instituteurs de Québec - , il ne manqua pas de flairer des visées d'intérêt personnel. Il s'en ouvrit à l'abbé Bois :

Les charlatans politiques, les riches avares et les ambitieux ont fait et font encore assez pour démoraliser le peuple complètement à l'égard de l'acte des écoles. Or il en est un sur les lieux et il en est qui sont à la tête de la presse, tels maîtres Turcot[te] et J. C. et encore J. C., dont la jonction fait Cauchon-Crémazie, opposés autrefois l'un à l'autre, coalisés aujourd'hui pour faire tomber la loi actuelle des écoles, et avec elle le surintendant, dont ils convoitent la place. Il n’y a pas à se dissimuler, chacun de dire: "Ote-toi que je m'y mette". Ils travaillent maintenant ensemble un projet de loi d'éducation qui leur offrira à chacun une case, s'il devient loi... Pour moi, je vous l'avoue bien sincèrement, je suis dégoûté, bien plus de la conduite de semblables gens que de

125 avril 1850.

133 avril 1851. 
l'opposition insensée que nos bons habitants ont été amenés à faire à la loi dans certaines parties du pays. Si je n'avais pas sacrifié ma profession pour la position que j'occupe depuis près de 9 ans, ou si les émoluments que j'ai eus ou d'autres ressources me laissaient quelque moyen de subsistance, je ferais très volontiers mes adieux aux opposants et leur laisserais le champ libre. ${ }^{14}$

Le projet de loi rédigé par Crémazie et mis à la disposition de Cauchon fut envoyé au ministère. Il n'y fut pas donné suite. On lui donna le nom de "bill louche", par allusion à la perversité de ses principes et à une difformité physique de ses auteurs. ${ }^{15}$

Des amis du Dr Meilleur, jugeant qu'il devrait être membre de la Législature, sinon pour piloter les mesures relatives à l'éducation, du moins pour pouvoir répondre à l'Opposition, le prièrent de se porter candidat aux élections de décembre 1851. Il répondit que la vie politique ne lui souriait guère, et qu'il n'aimait pas mendier des faveurs, quand ce serait des suffrages. ${ }^{16}$ Par contre, il accueillit favorablement un projet de l'abbé Bois de Ie faire nommer au Conseil légisiatif, où il aurait l'Ópposition en moins et l'inamovibilité en plus. Il fit même des suggestions pour le faire réussir : tactique à suivre, personnes à voir ou à faire travailler, laïcs et prêtres. Toutefois, seul l'intérêt de sa famille le portait à vouloir le succès de cette affaire, car, personnellement, il n'aspirait qu'à se retirer de la vie publique. Il écrivait à l'ami de Maskinongé:

Je vous dirai de plus que, découragé, fatigué, et je puis dire vieilli par le travail et de longues années de combat et de sollicitude, si j'avais le moyen de me retirer entièrement de la vie publique, je le ferais dès ce jour; je le ferais quand même, si une nombreuse famille ne dépendait de moi pour son pain quotidien. La seule ambition que j'aimerais à pouvoir servir serait celle de pouvoir vivre tranquille au milieu de ma petite chaumière. Mais le besoin de ma famille, peutêtre aussi celui qu'on peut nonobstant avoir encore de moi, me fera consentir à être instrument entre les mains d'honnêtes gens, pour le bien de la cause. ${ }^{17}$

Je suis dans un état d'hypocondrie insurmontable. Je vous souhaite bien sincèrement de n'en être jamais atteint. Ce sera une raison de plus de vous dévouer, comme vous le faites si généreusement, à la cause publique, et d'une manière bien efficace quoiqu'occulte. ${ }^{18}$

1413 mars 1851 .

1515 juin 1859 .

162 octobre 1851.

178 décembre 1851.

189 décembre 1851 . 
L'abbé Bois s'étant risqué à lui proposer de se rendre à Québec pour voir les ministères susceptibles d'être favorables à son projet, sa fierté se cabra, et il répondit:

Malgré tout ce que vous me dites de l'opportunité d'un voyage à Québec, malgré que véritablement vous êtes mon paraclet, je ne puis l'entreprendre sans y être appelé, ce à quoi je m'attends jusqu'à un certain degré. Je ne me suis jamais jeté dans les jambes des administrations, si ce n'est pour les opposer, modestement mais avec fermeté, lorsque j'ai cru qu'elles étaient disposées à faire des choses que je regardais comme contraires à l'éducation populaire. J'aurais peut-être pu me faire un chemin comme un autre, si j'avais été un peu complaisant envers ceux qui pouvaient tout faire pour moi, si j'eusse mis mon individualité à leur disposition pour leur servir d'instrument. J'en connais plus d'un qui n'ont obtenu titres et fortunes qu'à ces conditions. ${ }^{19}$

Le projet de l'abbé Bois échoua, et le Dr Meilleur retomba plus abattu que jamais. Presque toutes ses lettres sont remplies d'aveux navrants comme ceux-ci:

Je suis toujours souffrant, et surtout fatigué. Je me sens vieux. L'énergie mentale diminue avec l'énergie animale, de manière à ne me laisser que le quod justum de vie et de vigueur pour remplir tels quels les devoirs importants de ma charge. Je vous assure que si je pouvais trouver à me placer dans un fromage de Hollande, je le ferais sans hésiter. ${ }^{20}$

Mon mal de côté ne cesse de me tourmenter beaucoup. Je sens le besoin de me reposer, de me retirer même. Mais comment faire ? Je n'ai pas les moyens de le faire, et le Gouvernement n'a pas de sinécure à me donner. Je serais heureux s'il me donnait 1000 à 1500 arpents de bonne terre pour mes garçons, et pour les mettre en état de me soutenir sur mes vieux jours. Je me considère comme étant véritablement infirme, et si la lutte que j'ai encore à soutenir est longue et un peu orageuse, je succomberai inévitablement. J'ai trop combattu dans ma vie pour pouvoir le faire toujours impunément. Je le répète donc bien franchement, si j'avais un autre moyen d'existence, je me retirerais très volontiers et bientôt de la lutte. C'est la considération de ma famille qui me retient; car, pour moi, il me faudrait si peu pour vivre, que je le trouverais nonobstant. ${ }^{21}$

La maladie l'avait rendu soupçonneux. Il avait la manie de la persécution. Aussi fut-il pris de panique lorsque, au début

1921 janvier 1852.

206 mars 1852.

2129 mars 1852. 
de la session de 1853 , le Parlement nomma un comité spécial de neuf députés pour s'enquérir de l'état de l'instruction, du fonctionnement de la loi des écoles, de l'efficacité du département de l'Instruction et des moyens de rendre plus efficaces les dispositions législatives adoptées pour l'avancement de l'instruction dans le Bas-Canada. "Il est évident, écrivit-il, qu'il y a coalition contre les Inspecteurs et contre le Surintendant. On en veut à ces nouveaux fonctionnaires, parce qu'ils ne sont pas tous des créatures politiques et surtout parce qu'ils promettent succès aux amis de la cause et humiliation aux opposants." 22

Ce qui donnait un fondement à ses soupçons, c'est que la présidence du comité était confiée au député de Saint-Hyacinthe, Louis-Victor Sicotte. Ce dernier en avait contre le Dr Meilleur. Il croyait - à tort - que c'était le surintendant qui avait fait nommer inspecteur d'écoles pour le comté de Saint-Hyacinthe le $\mathrm{Dr}$ Consigny, son adversaire dans toutes les élections, et il avait été froissé d'une opinion contraire à la sienne donnée à cet inspecteur par le surintendant. Le Dr Meilleur s'attendait à une vengeance de la part de Sicotte, et, découragé, il songea à prendre le chemin de l'exil.

Voyez dans quelles mains je me trouve, lorsque cet homme est le président d'un comité d'enquête sur mon administration. C'est une véritable persécution sous l'apparence de l'intérêt que l'on porte à la cause de l'éducation... Dans une espèce de désespoir, à la vue des tourments qu'on me prépare, je regar de le chemin qui conduit aux Etats-Unis, où, pour proclamer la magnanimité du gouvernement et du pays que je sers fidèlement depuis 20 ans, j'errerai à l'aventure, suivi de partie au moins de ma famille dénuée de tout!! Oh! priez pour moi et pour elle. Elle ne comprend pas le sort auquel elle est exposée. Alphonse est en-bas, en ce moment, intriguant joyeusement ses sœurs, pendant que, sans quelques efforts pour ranimer mon peu de courage ébranlé, je couvrirais le reste de cette page de larmes. ${ }^{23}$

Une série de vingt-quatre questions fut adressée par le comité Sicotte à plus de quatre cents personnes: membres du clergé, secrétaires des municipalités scolaires, citoyens de marque. "Nul doute que cette série ne soit un système ouvert à l'accusation et à la délation", pensait Meilleur. ${ }^{24}$ C'était aussi l'opinion de l'évêque coadjuteur de Montréal, Mgr Joseph LaRocque. Celui-ci, en l'absence de Mgr Bourget, parti en Europe, adressa

2214 mars 1853.

234 avril 1853 .

24 Ibid. 
une circulaire à ses prêtres pour leur conseiller de ne rien répondre; mais elle leur parvint un peu tard: plusieurs avaient déjà répondu au questionnaire du comité. De son côté, Meilleur soumit son rapport annuel et ceux des inspecteurs. Il comptait beaucoup sur ces documents pour embarrasser le comité et se relever dans l'opinion publique. Aussi fut-il fortement contrarié en apprenant qu'ils ne seraient publiés que dans plusieurs mois, dans l'appendice du Journal de l'Assemblée législative, "que très peu de personnes reçoivent". ${ }^{25}$ Et lorsque, en novembre (1853), il prit connaissance du rapport du comité par le Journal de Québec, il fut révolté par le parti-pris qu'il y remarqua. "On a agi avec une industrie remarquable pour faire ressortir des réponses faites au Comité tout ce qui milite contre la loi, et surtout contre mon administration, on a défiguré et même soustrait tout ce qui pouvait militer en leur faveur." 26

La publication du rapport du comité Sicotte coïncidait avec une recrudescence de violence des journaux rouges ( Le Pays, Le Moniteur) contre le surintendant. On ne lui pardonnait pas d'avoir donné gain de cause aux Frères des Ecoles chrétiennes de Beauharnois contre l'inspecteur Magloire Lanctôt, qui avait voulu les chasser, et aux habitants de Saint-Constant de Laprairie contre le commissaire d'écoles Bruneau, qu'ils avaient démis parce que devenu protestant. On lui reprochait de suivre les directives des évêques voulant que les écoles mixtes fussent tenues autant que possible par des personnes de sexe féminin. "Le but avoué verbalement est de chasser les filles et les femmes des écoles, et surtout les Frères et les Sœurs, parce que le clergé a trop d'influence sur ces personnes. Et, comme on me croit favorable à ces personnes, on veut me chasser. C'est là l'autre but avoué." ${ }^{27} \mathrm{La}$ vue du danger auquel était exposée la cause de l'éducation le fit se raidir de nouveau.

Je serais plus disposé à me retirer si on ne me persécutait pas, si on ne faisait pas l'incroyable pour ruiner ma réputation et opérer ma chute... Ce serait lâcheté qu'abandonner le tout dans le moment présent. ${ }^{28} \mathrm{Si}$ je ne consultais que mon goût, je me retirerais de suite; mais la persécution me fait un devoir de demeurer en office jusqu'à ce que tout soit examiné et décidé; le bien de ma famille me fait aussi un devoir de patienter. ${ }^{29}$

2214 mars 1853.

2630 décembre 1853.

27 Mi-octobre 1853.

2830 décembre 1853.

294 janvier 1854. 
Je désire le repos, mon âge et mes infirmités m'en font un besoin; mais puisque je suis à l'affût pour une bonne cause, je suis disposé à combattre encore avec les gens de bien pour son triomphe. ${ }^{30}$

Sicotte voulait un Conseil de l'Instruction publique composé des inspecteurs d'écoles. Meilleur flaira le danger. A ses yeux, les inspecteurs d'écoles formés en conseil pourraient neutraliser, maîtriser et abattre le surintendant, et gouverner à leur guise ou suivant la volonté des hommes politiques dont ils seraient les créatures et les protégés. "Que ne feraient-ils pas aujourd'hui, à l'instigation de Sicotte et de Lanctôt, s'ils formaient le conseil de l'instruction publique, dont le surintendant aurait à choisir, ou d'être le commis, l'instrument obligé de leurs menées, ou d'abandonner le poste à l'un des leurs décidé à être le complaisant complice de tous leurs noirs projets". ${ }^{31}$

Pour déjouer ce plan, le Dr Meilleur élabora un projet de loi, aux termes duquel le surintendant serait de facto soit en le plaçant dans le Conseil législatif, soit autrement membre de l'Exécutif, avec droit d'y siéger lorsqu'il délibérerait sur toute mesure relative à l'instruction publique. L'Exécutif ne pourrait faire qu'une chose, seul: nommer le surintendant. Celui-ci une fois nommé, tout ce qui est du ressort de l'administration générale serait fait par lui ou par son entremise, et il ne consulterait le Conseil de l'Instruction publique que lorsqu'il le jugerait à propos, dans le cas de graves difficultés ou de mesures extraordinaires à prendre. ${ }^{32}$ Ce projet resta enfoui dans le bureau de l'Exécutif.

Un mois avant l'ouverture de la session, Meilleur envoya à ce même Exécutif son rapport pour l'année 1853. Il comptait sur ce rapport pour se justifier aux yeux du public des attaques des Rouges. Et, comme il s'attendait à ce qu'il ne fût pas imprimé, il eut la hardiesse de faire imprimer et distribuer aux membres de la Chambre un tableau statistique qui put suppléer à son absence. Son rapport lui-même ne fut même pas déposé devant la Chambre. Il faut dire que la session ne dura que dix jours - du 13 au 22 juin. Il n'en fut pas moins humilié.

Je n'aime pas à m'élever, surtout à me raidir contre l'autorité établie; mais je me sens humilié, lorsque je la vois indifférente, apathique, et même apparemment disposée à me faire subir

3019 janvier 1854.

3130 décembre 1853.

3219 janvier 1854. 
le joug. Chacun a sans doute son grain de vanité. Or la mienne, c'est de croire à mon mérite d'avoir fait autant sinon plus de bien à mon pays qu'aucun de nos charlatans politiques. Je le crois, parce que j'ai toujours agi honnêtement et sous l'influence des principes humanitaires et sous l'empire de la religion. ${ }^{33}$

Je soupçonne que tous ces gens-là me boudent... Puis, je crois que pour régner ils n'auraient pas de scrupule à me sacrifier, moi et mes propositions, à des exigences politiques. L'abandon dans lequel ils m'ont laissé, au milieu des coups que les rouges et autres ambitieux me lancent depuis deux ou trois ans, le prouve assez... Voyez-vous je ne suis pas l'instrument politique du gouvernement et je m'oppose souvent à ses propres mesures en fait d'instruction publique, à celles au moins que proposent ses amis ou ceux de ses opposants qu'il veut amadouer. J'ai raison de croire qu'ils sont fatigués de moi, et moi je suis profondément dégoûté d'eux. Mais comment faire ? Je suis nécessiteux, vieux et usé, et j'ai une nombreuse famille dont la plupart en bas âge dépend de moi pour les premiers soins de son existence... Oh ! qu'il est difficile à un homme ayant un caractère comme celui qui me domine de se soumettre soit au rôle humiliant de servile instrument d'hommes dédaigneux et arbitraires, ou à l'indigence. Si je n'avais que moi à penser, je ne les troublerais pas longtemps, mais ma famille... ${ }^{34}$

A la session de l'automne 1854, à l'instigation de Magloire Lanctôt, le député Charles Daoust demanda copie de la correspondance du Dr Meilleur au sujet des Frères de Beauharnois et des Sœurs de Longueuil. Meilleur vit dans cette démarche une tactique de ses ennemis pour arriver à le perdre.

Il est à craindre que le parti ministériel, soit par ignorance ou par apathie ne laisse faire les rouges comme ils ont laissé faire Sicotte. A moins qu'on attire l'attention des premiers sur les intentions des derniers, il $\mathrm{y}$ a tout à craindre pour moi... Si je succombe, je ne sais ce que je ferai, mais je crois que, repoussé, ou du moins abandonné par mon pays, j'irai demander du pain à l'étranger. ${ }^{35}$

$\mathrm{Si}$ je n'avais pas une nombreuse famille, mon choix serait bientôt fait, et j'aurais encore du plaisir à aller demander, en échange de quelques services mieux appréciés, mon pain à l'étranger. Mon impression est que je serai obligé de le faire. Je suis déjà vieux, et surtout fatigué, usé.36

3329 juin 1854.

3418 septembre 1854.

3527 septembre 1854.

3813 octobre 1854 . 
$\mathrm{Au}$ cours de cette même session, le député Luc-Hyacinthe Masson demanda qu'une copie du projet de loi du Dr Meilleur sur l'éducation primaire soit déposée devant la Chambre. Sa motion donna lieu à un débat quelque peu acrimonieux. Les ministres, qui étaient au courant des choses, dirent que ce projet ne faisait pas partie du rapport du surintendant et devait par conséquent être considéré comme une correspondance privée. Pour Cauchon, le projet devait être considéré comme celui qu'un ministre aurait fait et qui pouvait être mis de côté par suite d'une délibération avec ses collègues. Le Dr Meilleur craignit que la motion Masson ne contribuât à indisposer le ministère envers lui.

Autre raison pour lui de songer à sa retraite: il s'attendait à ce qu'on cherchât à faire dépendre son département d'un portefeuille ministériel.

J'ai déjà témoigné à quelques ministres que je ne voulais pas être un sujet d'embarras pour eux, que je suis prêt à me retirer, mais que je m'attendais, en toute justice, ou qu'on me donnera une pension ou une sinécure qui me permette une existence qui ne milite pas contre la réputation du pays, en laissant souffrir du nécessaire un de ses vieux serviteurs... J'ai été même jusqu'à désigner une place que je pourrais occuper; mais, véritablement, je suis si fatigué et dégoûté que la retraite est la seule chose que je désire, si on veut bien y attacher une existence convenable. ${ }^{37}$

Au mois de novembre (1854), le Parlement fit, à même le fonds des écoles primaires du Bas-Canada, des octrois spéciaux aux collèges du Bas-Canada et aux Grammar Schools du HautCanada. Meilleur en fut très mécontent. "C'est par suite de ce vol que j'ai envoyé ma résignation", écrira-t-il plus tard. ${ }^{38}$

Un remaniement ministériel eut lieu en janvier 1855. Chauveau perdit le poste de secrétaire provincial, qui échut à George-Etienne Cartier. Pour l'empêcher de se venger, on lui accorda ce qu'il convoitait, la surintendance de l'Education, dont il voulait porter le salaire à $£ 1000$ et établir le bureau à Québec, "sous le porche de l'Université Laval" ${ }^{39}$ Les ministres du district de Montréal sacrifièrent le Dr Meilleur, pour plaire à ceux du district de Québec. Sans l'intervention de Cartier, son ami, on l'aurait tout simplement destitué. Et comme on ne

3728 octobre 1854 .

3830 mai 1859 .

3931 janvier 1856. 
voulait pas lui accorder de pension, il dut accepter "avec une espèce de reconnaissance" 40 la place de directeur des Postes de la Cité de Montréal, devenue vacante par la retraite d'Alfred Larocque. ${ }^{41}$

Le 28 juin, il recevait l'ordre d'occuper son bureau dès le 1er juillet, ce qu'il fit, non sans maugréer contre son successeur. "Sur son ordre, les choses se préparent comme pour l'avènement d'un prince au trône." 42

La démission du Dr Meilleur est l'aboutissement de la lutte incessante qu'il a dû mener contre les éteignoirs fanatiques, les politiciens envieux et les Rouges laïcisants. Elle est le dénouement d'un drame intérieur où l'agressivité alternait avec le découragement. Elle serait survenue plus tôt, n'eut été la nécessité où il se trouvait de subvenir aux besoins de sa nombreuse famille. Il a lutté pour des principes: tenir la politique à l'écart du domaine de l'éducation et maintenir le caractère religieux de l'école. Il était soutenu par son tempérament, autant que par la fierté de son caractère. Mais il s'est épuisé, moralement comme physiquement. Il était devenu aigre, soupçonneux et - disons-le, puisque lui-même s'en était rendu compte embarrassant pour les ministres du Gouvernement. Hélas ! il ne trouva pas dans ses nouvelles fonctions le repos auquel il avait tant aspiré. L'avenir lui réservait d'autres déboires.

4031 janvier 1856.

41 Le titulaire était James Porteous. BRH, 42 (1936) : 517.

1217 juillet et 11 décembre 1855. 